\title{
A Breif Review of the Effects of Physical Activity in Subjects with Cardiovascular Disease: An Interpretative Key
}

\author{
Gaetano Altavilla', Francesca D’Elia² and Gaetano Raiola² \\ ${ }^{1}$ University of Split, Faculty of Kinesiology, Split, Croatia, ${ }^{2}$ University of Salerno, Department of Human, Philosophical and Education, Salerno, Italy
}

\begin{abstract}
Today is consolidated the importance of physical activity to health, so it is important to know the effects of exercise for primary prevention and secondary. A regular physical activity of mild intensity results in significant benefits in term of health, while a sedentary lifestyle and otherrisk factors contributeto development of chronic degenerative diseases, in particular cardiovascular. The advanced age does not contraindicate physical activity and exercise can prevent cardiovascular and disability diseases in the elderly. In the elderly person physical exerciseis able toimprove muscular tone and movement skills, of reduce the risk of sudden death caused by cardiovascular diseases, of reduce the development of tumors and metabolic disorders, as well as of delay the decline of cognitive function. The aim of this literature review is to clarify the link and the effects between physical activity, aging and cardiovascular diseases, providing practical indications for a useful physical activity for elderly subjects with cardiovascular problems;adapting to the skills of the elderly person, prescribed after careful assessment of the environmental conditions in which it must take place. In conclusion, a physical activity program finds a sure indication both in elderly subjects without and with pathologies and in the elderly with high risk of disability.
\end{abstract}

Key words: sedentariety, movement, benefits, aging, diseases cardiovasculary, personalized activity

\section{Introduction}

The evolution of lifestyles, along with the possibility of access to physical education from the social groups such as the elderly and children, have initiated a modification of perception and social meaning of the movement and physical activity, emphasizing the educational and social value and decreasing the competitive component (D’Isanto \& Di Tore, 2016). Practicing regular physical activity offers several physical and mental benefits for elderly (Gaetano, 2016), including slowing the aging process, promoting psychological and cognitive well-being, reductionof the risk of physical disability,increasing longevity and greater functional independence (American College of Sports Medicine, 2009). The education to the physical activity, motor and sports should be the center of the teaching-learning processes and help the process of construction of knowledge and skills within the school (Raiola, 2013). And how much this knowledge and skills system can be conditioned by social and cultural aspects (D'Isanto, Altavilla, \& Tafuri, 2017).Today is consolidated the importance of physical activity to health, so it is important to know the effects of exercise for primary prevention and secondary (Tiziana, Antonetta, \& Gaetano, 2017). It is not easy to separate the effects of aging from the decrease in physical activity linked to age. Regular physical activity of moderate intensity leads to significant health benefits in all age groups; moreover, a sedentary lifestyle contributes, together with other risk factors, to the development of numerous diseases, in particular those of the cardiovascular apparatus. Several studies reinforce the view that physical activity has favorable effects on the state of health (Paffenbarger \& Lee, 1997). In old age, physical activity is not contraindicated; on the contrary, it can prevent cardiovascular diseases and the disability of the elderly (Rengo, Leosco, \& Iacovoni, 2004). There are

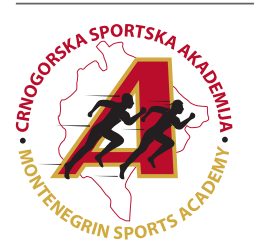

Correspondence:

G. Altavilla

University of Split, Faculty of Kinesiology, Teslina 6, Split, Croatia

E-mail: gaetano.altavilla_@libero.it 
many positive aspects of regular physical activity in the elderly

(Shekelle, Maglione, \& Mojica, 2003):

- reduction the risk of sudden death, heart attack or heart disease;

- reduction of up to $50 \%$ risk of developing colon cancer; - the reduction of the risk of up to $50 \%$ of the development of type 2 diabetes;

- prevention or reduction of hypertension;

- reduction of the risk of developing cognitive impairment and dementia;

- the reduction of symptoms of anxiety, stress and depression;

- weight loss and decreased risk of obesity, with benefits of $50 \%$ compared to those with a sedentary lifestyle.

A sedentary lifestyle can contribute together with other risk factors to the development of various chronic diseases, particularly those affecting the cardiovascular system. Therefore, the primary objective of public health should be the development of strategies to promote the spread of physical activity in all environments and in all age groups. The promotion of physical activity is becoming a priority public health action, often included in health plans and programs all over the world. In Italy, in the National Health Plan of 2011-2013, the aim was to increase on average $10 \%$ physical-sport activity in free time among the elderly, starting from the consideration that physical activity has a protective effect not only for cardio-respiratory and cardio-vascular pathologies, but also for osteoarticular and metabolic diseases. In the USA, the Healthy People 2010 program identifies physical activity as one of the main health objectives, such as the European Union in the Public Health Program (2003-2008) indicates programs that support and promote physical activity. The perform of regular physical activity can therefore play a fundamental role in improving the functional abilities of the elderly and their quality of life.

The aim of this literature review is to clarify the link and the effects between physical activity, aging and cardiovascular diseases, providing practical indications for a useful physical activity for elderly subjects with cardiovascular problems.

\section{Methods}

The review of the literature and scientific documents was conducted through the use of several databases: PubMed, MedLine, Google Scholar. Relevant bibliographies were sought to identify the effects of physical exercise in individuals with cardiovascular disease.

\section{Results}

Sedentary lifestyle is a cardiovascular risk factor

There are numerous evidences of the beneficial effects of physical activity in countering the reduction of cardiocirculatory performance (Hakim et al., 1999; Hambrecht et al., 2000; Donal et al, 2011). In sedentary subjects, in fact, there is a more marked reduction in systolic function and cardiac output, accompanied by an increase in heart rate; subjects who have undergone long-term sickness, develop a hypokinetic syndrome overlapping that of aging. It is widely known that a lifestyle characterized by physical inactivity is a cardiovascular risk factor. A sedentary lifestyle is responsible for a significant increase in cardiac morbidity and mortality. Physical exercise has beneficial effects on cardiovascular disease, as demonstrated by Paffenbarger et al. (1997), in a study conducted on
Harvard college students. This work has shown that a reduction in the risk of death in physically active subjects compared to sedentary ones. Furthermore, the authors showed that in the group of subjects who developed arterial hypertension and who carried out physical activity there was a reduction in mortality of $50 \%$. The benefits of the exercise were also evident in the assessment of the incidence of coronary heart disease independently of other factors and suggesting that moderate sporting activity is associated with the reduction of the mortality. The study showed that in order to achieve a reduction in mortality of $20 \%$, an exercise intensity is required that leads to an energy consumption of about 4200 KJules a week (equivalent to 30 minutes of exercise a day, for at least 4-5 days at week). An Inferior intensity were not sufficient to guarantee significant improvements in the prognosis. Physical activity of mild to moderate intensity has also shown benefits in elderly subjects (Wannamethee, Shaper, \& Walker, 1998), indicating the need for a specific public health program that encourages physical exercise in this increasingly prevalent age group.

\section{Effects of the physical exercise on the diseases cardiovasculary and suggestions}

In the Longitudinal Study of Aging, conducted on subjects over seventy years old, the importance of maintaining a good level of physical activity in old age is further confirmed, demonstrating an inverse relationship between physical activity and mortality (Rakowski \& Mor, 1992). The improvement of cardiac function is possible thanks to an increase in oxygen consumption and contractile capacity of the heart. Hasbeen shown that in elderly subjects with acute myocardial infarction, routine physical activity prior to the ischemic event favors both short-term and long-term survival (Rengo, Galasso, \& Piscione, 2007).Significant effects of exercise on the blood pressure have been reported in patients with mild or initial hypertension, where it is possible through physical activity to prevent or slow down the evolution towards stable arterial hypertension. Some studies (Kokkinos, Narayan, \& Colleran, 1995; Kokkinos\& Myers, 2010) have shown that regular exercise in subjects with mild or moderate arterial hypertension induces a mean reduction in blood pressure in the following measure: $8-10 \mathrm{mmHg}$ for maximum pressure and 7-8 $\mathrm{mmHg}$ for the minimum pressure. Therefore, it is advisable to use only aerobic running, favoring long distances, while avoiding repetitions (especially short ones), fartlek or training with net changes of pace (for example, of cross-country races). Aerobic training of moderate intensity, as well as the total duration of physical activity, play an important role in improving the HDL cholesterol and the elimination of the sedentariness could lead to a reduction of $15-39 \%$ of cardiovascular diseases, $33 \%$ of strokes and $22-33 \%$ of tumors (Sunami, Motoyama, \& Kinoshita, 1999). A review of primary prevention in women shows that even walking an hour a week has a protective effect on the risk of death from cardiovascular disease (Oguma, Sesso, Paffenbarger, \& Lee, 2002). Other reviews have shown that patients with cardiovascular disease benefit from a physical activity that consumes $1600 \mathrm{kcal}$ per week to counter the progression of coronary heart disease and $2200 \mathrm{kcal}$ per week for heart disease (Franklin \& Swain, 2003). It is important to remember that a physical exercise, preferably aerobic, must be mild-moderate: 30 minutes of movement (walking, swimming, cycling, etc.) are sufficient a day, at least five times a week, to obtain benefits. The screening should be planned 
for all seniors who wish to undertake a regular physical activity, both to identify subjects with chronic illnesses, symptoms linked at some diseases or to prescribe a program of appropriate and personalized exercises. The training must be practiced for several days a week and continued with a certain periodicity until becoming permanent. In general, the success of a program depends not only on the functional possibilities of the various interested parties, but also on the characteristics, intensity, duration of the exercise, its continuity and the repetition of the movements (Carlson, Ostir, \& Black, 1999). In order to maximize these benefits and reduce possible risks, we recommend monitoring the heart rate by increasing the intensity of resistance exercises, using $60-70 \%$ of the maximum heart rate for that subject as a goal. The maximal heart rate defined by the equation is considered: HRmax=208-0.7×age, HRmax is predicted, primarily, by age alone and is independent of sex and physical activity (Tanaka, Monahan, \& Seals, 2001).The decrease in resting heart rate is usually significant in the trained subjects and indicates the improvement of cardiovascular conditions. For a better and personalized definition of the maximal heart rate and to rule out any latent cardiovascular pathologies, it is always advisable, in the elderly subject, to perform an exercise electrocardiogram before planning a physical activity. Aging alters body composition and reduces its functionality and, consequently, increases the risk of disability and chronic diseases such as cardiovascular diseases, type 2 diabetes, obesity and certain types of cancer (Singh, 2004). Scientific evidence (Spirduso, Francis, \& MacRae, 2005) confirm that regular physical activity can reduce the physio- logical effects of aging and a sedentary lifestyle amplifies the effects of aging, while an active lifestyle prolongs the expectation of life and limiting the progression of chronic diseases and disabling conditions.

Suggested physical activity for healthy subjects and with cardiovascular diseases

Walking is the most used activity, but for those who have orthopedic and/or excess weight problems, water or bike activities are more recommended. For healthy elderly there are no contraindications to the cardiovascular efficiency training, which can lead to improvements even higher than $20 \%$ (Huang, Shi, Davis-Brezette, \& Osness, 2005) and in any case sensitive improvements already after the first three months of training. Many of the people suffer of problems at the cardiovascular system, naturally before being able to training subjects with these particular types of pathologies it is essential that the doctor gives the authorization and directives for the workouts. Regular aerobic activity is able to lower the heart rate and resting blood pressure, this allows the heart to reduce the workload and be less exposed to risks of overload diseases. Increasing the aerobic capacity of a person, improves the standard of living by providing more energy for everyday activities (American Association of Cardiovascular and Pulmonary Rehabilitation, 2004). In order to see the benefits, the training must at least have a duration of 12 weeks and must be practiced for at least two to three sessions a week. The duration of a single workout is at least 30 minutes of continuous exercise or accumulated with a heart rate at the beginning of the program

Table 1. Progression for intermittent aerobic exercise for people with normal functional capacity

\begin{tabular}{ccccc}
\hline Week & $\%$ Heart rate & $\begin{array}{c}\text { Round } \\
(\mathbf{m i n})\end{array}$ & $\begin{array}{c}\text { Repose } \\
(\text { min) }\end{array}$ & Repetitions \\
\hline $1-2$ & $50-60$ & $3-10$ & $2-5$ & $3-4$ \\
$3-4$ & $60-70$ & $10-20$ & optional & 2 \\
\hline
\end{tabular}

of at least 10 beats below that recommended by the cardiologist (ACSM's, 2013). The training of people with stable cardiovascular diseases must be authorized by a doctor, the role of the coach is to develop a program in line with the guidelines of the cardiologist without compromising the basic cardio-circulatory functions. Tables 1 and 2 compares and illustrates two

Table 2. Progression for intermittent aerobic exercise for people with low functional capacity

\begin{tabular}{ccccc}
\hline Week & $\%$ Heart rate & $\begin{array}{c}\text { Round } \\
(\mathbf{m i n})\end{array}$ & $\begin{array}{c}\text { Repose } \\
(\mathbf{m i n})\end{array}$ & Repetitions \\
\hline $1-2$ & $40-50$ & $3-10$ & $2-5$ & $3-4$ \\
$3-4$ & $50-60$ & $10-20$ & 2 & 2 \\
5 & $60-70$ & $10-20$ & 2 & 2 \\
\hline
\end{tabular}

training protocols for heart patients and healthy people; while in Tables 3 and 4 two different activities are suggested for two subjects with different cardiovascular pathologies.
In Tables 3 and 4 two different activities are suggested for two subjects with different cardiovascular pathologies.

Table 3. Examples of dynamic physical activities for a patient with limited cardiovascular capacity of 60 year

Constant cardiovascular commitment and moderate intensity mild

Walk in the flat 3-4 km/h
Climb the stairs 20 steps in $20 \mathrm{sec}$
Go out and walk with the dog $3-4 \mathrm{~km} / \mathrm{h}$
Pedaling in the plains $<12 \mathrm{~km} / \mathrm{h}$


Table 4. Example of activity for a 50-year low risk heart patient

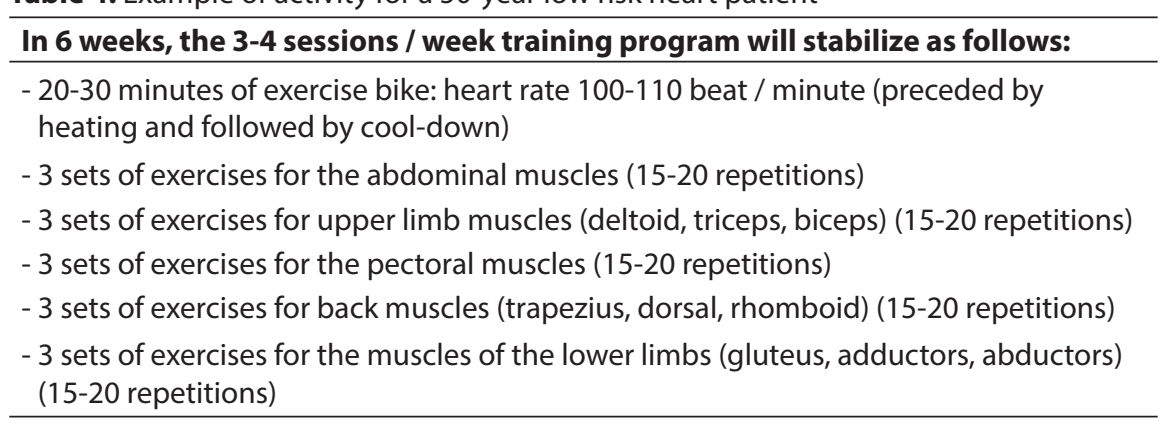

\section{Discussion}

The evolution of lifestyles, along with the possibility of access to physical education from the social groups such as the elderly and children, have initiated a modification of perception and social meaning of the movement and physical activity. Performing regular physical activity has beneficial effects on most organs and systems and is therefore essential for the primary prevention of a large number of diseases. From the evidences in the literature, contained in the present review, the physical exercise in older people produces three main benefits:

- improves functional capacity both in the healthy subject and in the patient with illness;

- is of supportive in widespread diseases such as arterial hypertension, diabetes and obesity;

- reduces the risk of developing chronic disabling diseases.

Although the beneficial effects of physical exercise increase with increasing frequency and intensity of activity, it is important to remember that the greatest benefits are due to moderate physical activity, as can be deduced from the various works mentioned in this document of review. A complete program, which integrates aerobic and anti-resistance activities, finds a sure indication in elderly subjects with and without pathologies or at high risk of disability. In conclusion, we can affirm that physical activity must be adequate to the subject's ability (organic, psychic, functional, etc.); be prescribed after correctly establishing intensity, duration and rhythm of repetition of the exercises and be congenial and suitable for the cultural, creative and emotional possibilities of the elderly person.

\section{Acknowledgements}

There are no acknowledgements.

\section{Conflict of Interest}

The authors declare that there are no conflicts of interest.

Received: 11 July 2018| Accepted: 26 August 2018|Published: 01 October 2018

\section{References}

ACSM's. (2013). Guidelines for Exercise Testing and Prescription, ninth edition, LWW.

American Association of Cardiovascular and Pulmonary Rehabilitation. (2014). Guidelines for Cardiac Rehabilitation and Secondary Prevention Programs. 4th ed. Champaign (IL): Human Kinetics.

American College of Sports Medicine. (2009). American College of Sports Medicine position stand: Exercise and physical activity for older adults. Medicine \& Science in Sports \& Exercise, 41, 1510-1530.

Carlson, J.E., Ostir, G.V., \& Black, S.A. (1999). Disability in older adults. 2: Physical activity as prevention. Behav Med; 24, 157-68.

Donal, E., Rozoy, T., Kervio, G., Schnell, F., Mabo, P., \& Carré, F. (2011) Comparison of the heart function adaptation in trained and sedentary men after 50 and before 35 years of age. Am J Cardiol., 108(7), 1029-37.

D'Isanto, T., Altavilla, G., \& Tafuri, D. (2017). Physical and sport activity and the relation to the eight key competences of the recommendation of the European Parliament and of the council. Sport Science, 10(1), 66-70.

D'Isanto, T. \& Di Tore, P.A., (2016). Physical activity and social inclusion at school: A paradigm change. Journal of Physical Education and Sport, 16(2), 1099-1102.

Franklin, B.A. \& Swain, D.P. (2003). New insights in the prescription of exercise for coronary patients. J Cardiovasc Nurs, 18, 116-23.

Gaetano, A. (2016). Relationship between physical inactivity and effects on individual health status. Journal of Physical Education and Sport, 16(Suppl. 2), 1069-1074.

Hakim, A.A., Curb, J.D., Petrovitch, H., Rodriguez, B.L., Yano, K., Ross, G.W. White, L.R., \& Abbott, R.D. (1999). Effects of walking on coronary heart disease in helderly men: The Honolulu Heart Program. Circulation, 100, 9-13.

Hambrecht, R., Gielen, S., Linke, A., Fiehn, E., Yu, J., Walthr, C., Schoene, N., \& Shuler, G. (2000). Effects of exercise training on left ventricular function and peripheral resistance in patients with chronic heart failure. JAMA, 283, 3095-3101.

Huang, G., Shi, X., Davis-Brezette, J.A., \& Osness, W.H. (2005). Resting heart rate changes after endurance training in older adults: a meta analysis. Med Sci Sports Exerc., 37(8), 1381-6.

Kokkinos, P.F., Narayan, P., \& Colleran, J.A. (1995). Effects of regular exercise on blood pressure and left ventricular hypertrophy in african-american men with severe hypertension. NEngl J Med; 30, 1462-7.

Kokkinos, P. \& Myers, J. (2010). Exercise and physical activity: clinical outcomes and applications. Circulation, 122, 1637-1648.

Oguma, Y., Sesso, H.D., Paffenbarger, R.S., \& Lee, I.M. (2002). Physical activity and all cause mortality in women: a review of the evidence. $\mathrm{Br} J$ Sports Med, 36, 162-72.

Paffenbarger, R.S. \& Lee, I.M. (1997).Intensityof physical activity related to incidence of hypertension and allcause mortality: an epidemiological view. Blood Press Monit, 2, 115-23.

Rakowski, W. \& Mor, V. (1992). The associationofphysical activity with mortality among older adults in the Longitudinal Study of Aging (1984-1988). J Gerontol Med Sci, 47, M122-9.

Raiola, G. (2013). Body knowledge and motor skills. Knowledge Cultures, 1(6), 64-72.

Rengo, F., Leosco, D., \& lacovoni, A. (2004). Epidemiology and risk factors for heart failure in the elderly. Ital Heart J, 5(Suppl 10), 9-16.

Rengo, G., Galasso, G., \& Piscione, F. (2007). An active life-style improves outcome of primary angioplasty in elderly patients with acute myocardial infarction. Am Heart J., 154, 352-60.

Singh, M. (2004). Exercise and aging. Clin Geriatr Med., 20, 201-21.

Spirduso, W.W., Francis, K.L., \& MacRae, P.G. (2005). Physical dimensions of aging (s ${ }^{\text {nd }}$ ed). Champaign, IL, Human Kinetics.

Tanaka, H., Monahan, K.D., \& Seals, D.R. (2001). Age-predicted maximal heart rate revisited, Journal of the American College of Cardiology, 37(1), 153-6.

Shekelle, P.G., Maglione, M., \& Mojica, W. (2003). Exercise programs for older adults: a systematic review and meta-analysis. Baltimore, MD: Centers for Medicare and Med-icaid Services - RAND Corporation reprint series.

Sunami, Y., Motoyama, M., \& Kinoshita, F. (1999). Effects of low- intensity aerobic training on the highdensity lipoprotein cholesterol concentration in healthy elderly subjects. Metabolism, 48, 984-8.

Tiziana, D., Antonetta, M., \& Gaetano, A. (2017). Health and physical activity. Sport Science, 10(1), 100-105.

Wannamethee, S., Shaper, A.G., \& Walker, M. (1998). Changes in physical activity, mortality, and incidence of coronary heart disease in older men. Lancet, 351, 1603-8. 